\title{
educação

\section{Avaliação de programas de intervenção para a aprendizagem socioemocional do professor: Uma revisão integrativa}

\author{
Alcione Moreira Marques', Luiza Hiromi Tanaka", \\ \& Adriana QueIróz Botelho Fóz $Z^{\text {III }}$
}

Universidade Federal de São Paulo, Brasil

\begin{abstract}
A profissão docente gera grande demanda emocional, o que pode afetar o bem-estar do professor e gerar problemas de saúde. As competências socioemocionais envolvem um conjunto de habilidades, conhecimentos e atitudes para reconhecer e lidar melhor com as próprias emoções e com a dimensão emocional dos alunos, o que pode diminuir o estresse e aumentar a qualidade da prática do professor. A aprendizagem socioemocional pode aumentar os recursos internos e a competência para lidar melhor com as demandas profissionais. Nesta revisão integrativa da literatura buscou-se levantar nas principais bases de dados mundiais (SCOPUS, PubMed, ERIC, SciELO, Web of Science, Campbell Collaboration) os estudos realizados sobre programas de intervenção específicos para desenvolver as competências socioemocionais do professor do ensino básico, suas características e impactos ou efeitos no professor. Foram incluídos 18 artigos sobre estudos que tiveram como objetivo a aprendizagem socioemocional do próprio professor, não associados a programas voltados aos alunos. Concluiu-se que embora haja poucos estudos sobre o tema, todos apresentaram impactos ou efeitos positivos no aumento de habilidades socioemocionais, na saúde, bem-estar, ou na eficácia docente.
\end{abstract}

Palavras-chave: Professor; Aprendizagem socioemocional; Estresse; Revisão integrativa

\section{INTRODUÇÃO}

Por décadas, a profissão do professor tem sido caracterizada como uma atividade que gera grande demanda emocional e que é potencialmente frustrante (Fisher, 2011). A profissão de professor é considerada pela Organização Internacional do Trabalho (OIT) como uma das mais estressantes. Independentemente do nível de ensino ou do tipo de escola, pública ou privada, a profissão de professor tem se configurado em alvo de diversos estressores. Os professores têm sido publicamente cobrados pelos fracassos na educação e raramente reconhecidos pelos seus sucessos (Carlotto, 2011).

O estresse profissional ou ocupacional pode ser entendido como o resultado do desequilíbrio entre a interação das condições de trabalho e as características individuais, de 
forma que as exigências apresentadas superam a capacidade de enfrentamento do indivíduo (Feuerhahn, Bellingrath, \& Kudielka, 2013). $\mathrm{Na}$ avaliação entre as exigências do trabalho e os recursos individuais disponíveis, três dimensões distintas podem ser consideradas: a emocional, a cognitiva e a física, tanto para as exigências quanto para os recursos dos quais o indivíduo dispõe (Feuerhahn et al., 2013). O estresse profissional pode se dar em uma ou mais destas dimensões, em razão do desequilíbrio destas interações, sendo as demandas emocionais especialmente críticas para o professor (Feuerhahn et al., 2013). Elas acontecem na interação social negativa que pode ocorrer no ambiente escolar, como, por exemplo, a crítica dos pais ou a ofensa verbal de alunos. Também a dissonância emocional, caracterizada pela exigência de se expressar uma emoção que não é genuinamente sentida numa certa situação, impõe ao docente uma grande exigência emocional (Feuerhahn et al., 2013). Este é um fenômeno que apresenta um caráter epidêmico mundial (Carlotto, 2011).

$\mathrm{O}$ alto nível de estresse laboral pode estar associado ao número de horas de trabalho, à pressão decorrente de muitas tarefas para o tempo disponível e à demanda emocional (Gomes \& Quintão, 2011). Pode levar ao esgotamento e a um crônico descontentamento com a atividade profissional, que caracterizam a Síndrome de burnout. O conceito de burnout teve sua origem nos Estados Unidos na década de 1970 e é um fenômeno psicológico que resulta da exposição prolongada ao estresse na atividade profissional (Gomes \& Quintão, 2011). Está associado à exaustão emocional, despersonalização, no sentido de indiferença e atitude distante associada ao trabalho, e baixa realização profissional (Maslach, Schaufeli, \& Leiter, 2001) e tem maior incidência nas profissões que envolvem atividades de cuidado e atenção direta (Steinhardt, Smith Jaggars,
Faulk, \& Gloria, 2011).

Áreas profissionais que antes não eram consideradas de risco por serem entendidas como vocacionais, entre elas a docência, mostraram-se com alta incidência de risco entre seus trabalhadores (Carlotto, 2011). Estudos indicam que em torno de um terço dos professores pode encontrar-se neste estado (Steinhardt et al., 2011), sendo a profissão que apresenta, mundialmente, a maior vulnerabilidade para manifestar a Síndrome de burnout (Fernet, Guay, Senécal, \& Austin, 2012). Do mesmo modo, o burnout aumenta a irritabilidade e a tensão do professor (Carlotto, 2011), com impactos deletérios na qualidade das relações interpessoais e no clima da organização escolar. O professor pode perceber as demandas do ambiente como ameaçadoras e, uma vez que não haja recursos suficientes para lidar com as mesmas, desenvolver estratégias defensivas como um modo de escape. Assim, um professor empático ou responsivo em relação aos estudantes pode, frente a contínuas experiências frustrantes, tornar-se apático, cínico e distante (Steinhardt et al., 2011). O prolongamento deste quadro pode gerar no professor problemas de saúde física e mental, podendo levar ao afastamento do trabalho (Carlotto, 2011).

Além dos impactos negativos para si, o estresse do docente pode gerar prejuízos para o discente. A diminuição do comprometimento com a atividade profissional afeta diretamente a atuação do docente e pode se converter em diferentes comportamentos que levam à retirada do ambiente profissional, como o alto índice de absenteísmo. Sentimentos como a insatisfação e a desmotivação podem diminuir a qualidade da atividade que o professor desenvolve em sala de aula e comprometer o desenvolvimento acadêmico dos alunos (Gomes \& Quintão, 2011). Embora seja essencial e desejável criar condições mais 
favoráveis ao trabalho no ambiente escolar, no que se refere a políticas educacionais, valorização da docência, melhor formação e infraestrutura, situações estressantes no cotidiano do professor são inevitáveis e continuarão fazendo parte de seu trabalho.

Se o exercício da docência se caracteriza, globalmente, como uma das profissões mais desgastantes, mostra-se relevante pensar em estratégias efetivas que reforcem os recursos do professor para lidar com as demandas de sua atividade laboral e que possam favorecer o aumento do seu bem-estar psicológico. Assim, é essencial que o docente desenvolva habilidades para lidar com situações potencialmente desafiadoras de modo mais positivo e eficiente (Gómez-Gascón et al., 2013). Na área da educação, tradicionalmente, as emoções foram consideradas um fenômeno mental menos importante que a racionalidade ou que se encontravam separadas do pensamento racional, resquício do pensamento cartesiano (Damásio, 2012). Esta concepção pode explicar, em parte, a falta de abordagem do tema nos cursos de formação pedagógica. Dessa maneira, o professor percebe-se despreparado para lidar com as próprias emoções e as dos estudantes.

Maior conhecimento sobre as emoções e mais habilidades para lidar com as mesmas podem promover a saúde emocional, diretamente relacionada ao bem-estar e à saúde mental (Karimzadeh, Salehi, Embi, Nasiri, \& Shojaee, 2014). Saúde emocional e mental vai muito além de simplesmente estar livre de transtornos; é o bem-estar psicológico amplo que envolve o modo como o indivíduo se sente sobre si mesmo, a qualidade de suas relações, as habilidades de manejar com seus sentimentos e com os estresses naturais da vida.

Ampliar o conhecimento sobre a dimensão emocional, aumentar a percepção de suas próprias reações e desenvolver melhores formas de lidar com as situações difíceis podem diminuir as reações de estresse (Gómez-Gascón et al., 2013). A melhor compreensão sobre as emoções, sua relação com a cognição, seu impacto na saúde física e mental se mostram relevantes para a atuação do professor frente às exigências de sua atividade profissional. Entender e lidar melhor com as emoções estão relacionados a algumas competências que não são, necessariamente, adquiridas de modo natural e podem ser construídas com suporte adequado (Jones, Bouffard, \& Weissbourd, 2013). Ou seja, habilidades socioemocionais podem ser aprendidas ou aprimoradas intencionalmente, aumentando os recursos internos do professor para exercer a docência.

A competência socioemocional compreende um conjunto de termos para uma ampla gama de habilidades que envolvem a inteligência emocional, competência social e autorregulação, abrangendo as áreas relacionadas aos processos emocionais, às habilidades interpessoais e à regulação cognitiva. Não é simplesmente atitude, interesse vocacional ou personalidade. A competência socioemocional está relacionada a uma inter-relação de habilidades emocionais e sociais que auxiliam a pessoa a manejar melhor com as próprias emoções, a relacionarse positivamente com outros, a executar tarefas diversas (estudar, trabalhar, etc.) e a lidar com as demandas diárias de uma maneira competente (Jones et al., 2013). São habilidades para a vida (Karimzadeh et al., 2014).

Tais competências, fundamentais para o desenvolvimento e sucesso pessoal, referem-se a pensamentos, sentimentos e comportamentos que podem ser agrupados em cinco aspectos centrais (Collaborative for Academic, Social, Emotional Learning - CASEL, 2019): autoconhecimento, consciência social, tomada de decisões responsável, habilidade 
de relacionamento e autocontrole. Cabe aqui diferenciar alguns termos relacionados às competências socioemocionais que são frequentemente utilizados como sinônimos, mas que têm significados distintos: aprendizagem, habilidades e competências socioemocionais.

Deacordo comaCASEL (2019), a competência socioemocional é atingida quando um indivíduo é capaz de combinar conhecimentos, habilidades e atitudes para atuar efetivamente nos campos pessoal, profissional, social e acadêmico. Este conceito coaduna-se com a abordagem de Dutra, Hipólito, e Silva (2000), que propõe que a competência se manifesta na ação, quando o indivíduo é capaz, frente a uma demanda, de mobilizar habilidades e conhecimentos em diferentes contextos (Dutra et al., 2000). A aprendizagem socioemocional (ASE) envolve o processo pelo qual se adquire tais conhecimentos, atitudes e habilidades para que seja atingido um nível satisfatório de competência socioemocional (CASEL, 2019). Deste modo, quando se fala sobre ações para desenvolver as competências socioemocionais do professor, tal refere-se ao processo de aprendizagem socioemocional. Já as habilidades socioemocionais são múltiplas (Marin, Silva, Andrade, Bernardes, \& Fava, 2017) e relacionam-se com o saber-fazer, a partir de uma série de procedimentos mentais que um indivíduo mobiliza para resolver um problema real (Bonotto \& Felicetti, 2014). Assim, as diversas habilidades, quando são utilizadas por adequação ao contexto, para atender às demandas que emergem de uma situação, constituem a competência socioemocional (Marin et al., 2017).

Os professores com mais competência socioemocional têm maior capacidade de regular suas emoções, são mais propensos a se sentirem satisfeitos com seu trabalho (Jones et al., 2013) e estabelecem relações melhores no ambiente escolar. É importante ressaltar que estudos associaram a qualidade do relacionamento entre o professor e o aluno e a motivação deste último com a aprendizagem (Maulana, Opdenakker, \& Bosker, 2014), o que, por sua vez, tem reflexos no desempenho acadêmico (Jones et al., 2013; Maulana, Opdenakker, Stroet, \& Bosker, 2013). Além disso, as competências socioemocionais do professor o tornam um modelo positivo e influenciam sua capacidade de desenvolver estas mesmas competências dos alunos (Jennings \& Greenberg, 2009). Embora a ASE possa ser estimulada de maneira informal, ela é mais eficaz quando sistematizada e disseminada através de programas ou intervenções estruturadas.

Estes aspectos ampliam a relevância de programas de intervenção para a aprendizagem socioemocional (PASE) no desenvolvimento da competência socioemocional dos educadores como forma de impactar positivamente a educação como um todo. Embora tenham se tornado cada vez mais presentes intervenções para o desenvolvimento de habilidades socioemocionais dos alunos, ainda são poucos os que se voltam para a aprendizagem socioemocional dos professores.

Esta pesquisa de revisão integrativa procurou levantar os artigos sobre programas de intervenção para a aprendizagem socioemocional voltados para os professores de modo a comparar, contrastar e analisar seus diversos aspectos, trazendo à luz os efeitos e impactos para os professores participantes.

\section{METODOLOGIA}

A revisão integrativa da literatura é uma metodologia que busca sintetizar informações sobre um tema específico, permitindo a inclusão de estudos diversos para a compreensão do fenômeno analisado (D’Avila, Pinto, Hauser, Gonçalves, \& Harzheim, 2017). 
É um estudo descritivo que contribui para evidenciar o conhecimento atual sobre uma temática específica, já que é elaborada com o objetivo de sintetizar, comparar, analisar e discutir estudos independentes sobre um mesmo assunto (Souza, Silva, \& Carvalho, 2010). Há critérios claros para a busca e seleção dos estudos que balizam a inclusão e a exclusão dos mesmos e que devem ser explicitados pelo pesquisador de forma clara (D'Avila et al., 2017). Foram seguidas as seguintes etapas: (1) elaboração da pergunta de pesquisa; (2) busca nas bases de dados; (3) seleção dos artigos; (4) extração dos dados; (5) síntese dos dados; (6) análise dos dados; (7) publicação dos dados (Souza et al., 2010).

Para a revisão integrativa, formulouse a seguinte questão: quantas e quais são as intervenções estruturadas para a aprendizagem socioemocional de professores? Utilizaram-se os seguintes descritores: professor, socioemocional ou inteligência emocional, programa ou intervenção, e escola. Não foi delimitado período, idioma ou país de publicação.

Para esta revisão integrativa foram consultadas as bases de dados públicas PubMed (National Library of Medicine, Estados Unidos), ERIC (Education Resources Information Center, do Institute of Education Science, Estados Unidos), SCOPUS (Elsevier, Holanda), Web of Science (Clarivate Analytics, Estados Unidos), SciELO (Scientific Eletronic Library Online) e Campbell Collaboration (vinculada a Cochrane Collaboration). Buscou-se identificar artigos científicos sobre a aplicação de programas de intervenção estruturados para a aprendizagem socioemocional (PASE) de professores da escola regular, com o objetivo de desenvolver ou aprimorar as habilidades socioemocionais do docente da escola de ensino básico regular.

Foram excluídas teses, dissertações e livros. Excluiu-se também artigos que somente descreveram ou discutiram os fundamentos / as características dos PASE, bem como aqueles voltados para docentes de instituições de ensino que não o básico regular, como escolas especiais, universidades ou escolas técnicas. Foram igualmente excluídos estudos sobre programas de intervenção não universais para o desenvolvimento de habilidades socioemocionais, ou seja, voltados para situações específicas dentro da escola.

A coleta identificou 398 artigos científicos sobre a aplicação de programas de intervenção universais em aprendizagem socioemocional em escolas básicas regulares. Após a leitura dos títulos, resumos, e de alguns artigos na íntegra, foram selecionados 18 artigos que tiveram como objetivo avaliar os impactos ou efeitos de programas específicos para o desenvolvimento de habilidades socioemocionais do professor de maneira exclusiva ou em conjunto com outros sujeitos, apresentados na Tabela 1.

\section{RESULTADOS}

Esta revisão integrativa da literatura identificou 398 artigos relacionados a programas de intervenção em aprendizagem socioemocional aplicados na escola regular. Destes, 334 artigos (84\%) tratam da aprendizagem socioemocional dos alunos e 108 (27\%) envolvem também professores ou exclusivamente estes. Destes 108 estudos que incluíram o professor de algum modo, parte trata do treinamento ou implementação, pelo professor, de programas, na escola, para o ensino das competências socioemocionais aos alunos (32 artigos - 8\%). Embora não sejam pesquisas sobre intervenções especificamente voltadas para o desenvolvimento destas competências do professor, a maioria destes estudos explora os impactos da participação do professor no treinamento e na aplicação dos programas (Martinsone \& Damberga, 2017). 
TAB ELA 1

Resultado da Revisão Integrativa: Programas Específicos para ASE do Professor

\begin{tabular}{|c|c|c|c|c|c|c|}
\hline $\begin{array}{l}\mathrm{N}^{\circ} \\
\text { Estudo }\end{array}$ & Autores/Ano & $\begin{array}{l}\text { Participantes } \\
\end{array}$ & Temática & $\begin{array}{l}\text { Tipo de } \\
\text { intervenção }\end{array}$ & Resultados & $\begin{array}{l}\text { Descrição do } \\
\text { programa/ } \\
\text { intervenção }\end{array}$ \\
\hline 1 & $\begin{array}{l}\text { Jennings, Foltz, } \\
\text { Snowberg, Sim, } \\
\text { \& Kemeny } \\
(2011)\end{array}$ & 56 docentes & $\begin{array}{l}\text { Dois estudos que } \\
\text { avaliaram o programa } \\
\text { Cultivating } \\
\text { Emotional balance } \\
\text { Training }\end{array}$ & $\begin{array}{l}\text { Programa de ASE } \\
\text { apoiado em } \\
\text { Mindfulness }\end{array}$ & \begin{tabular}{|l|} 
Um estudo mostrou \\
melhora do clima da sala \\
de aula, bem-estar e \\
atitudes frente a alunos \\
desafiadores. O segundo \\
estudo não apresentou \\
efeitos significativos
\end{tabular} & $\begin{array}{l}8 \text { semanas (4 } \\
\text { tardes e } 4 \text { dias } \\
\text { inteiros) }\end{array}$ \\
\hline 2 & \begin{tabular}{|l|} 
Jennings, \\
Snowberg, \\
Coccia, \& \\
Greenberg \\
(2011)
\end{tabular} & 74 docentes & $\begin{array}{l}\text { Estudo piloto com } \\
\text { duas escolas para } \\
\text { avaliar o programa } \\
\text { CARE para ASE }\end{array}$ & $\begin{array}{l}\text { Programa de ASE } \\
\text { apoiado em } \\
\text { Mindfulness }\end{array}$ & \begin{tabular}{|l|} 
Redução de estresse e \\
melhora da performance \\
mais significativo com \\
professores de ambientes \\
de maior risco
\end{tabular} & $\begin{array}{l}5 \text { dias de } 6 \text { horas } \\
\text { ( } 30 \text { horas), ao } \\
\text { longo de } 3 \\
\text { meses, com } \\
\text { telefonemas de } \\
\text { coaching entre as } \\
\text { sessões e um } \\
\text { reforço ao final }\end{array}$ \\
\hline 3 & $\begin{array}{l}\text { Karimzadeh, } \\
\text { Goodarzi, \& } \\
\text { Rezaei (2012) }\end{array}$ & 76 docentes & $\begin{array}{l}\text { Avaliou os efeitos do } \\
\text { programa EIT de } \\
\text { ASE }\end{array}$ & Programa de ASE & $\begin{array}{l}\text { Aumento das habilidades } \\
\text { socioemocionais. } \\
\text { Correlação entre } \\
\text { habilidades emocionais e } \\
\text { saúde geral }\end{array}$ & $\begin{array}{l}10 \text { sessões } \\
\text { semanais de } 2 \\
\text { horas }\end{array}$ \\
\hline 4 & $\begin{array}{l}\text { Pérez-Escoda, } \\
\text { Filella, Alegre, } \\
\& \text { Bisquerra } \\
(2012)\end{array}$ & $\begin{array}{l}92 \text { docentes } \\
\text { e } 423 \text { alunos }\end{array}$ & $\begin{array}{l}\text { Avaliou dois } \\
\text { programas de ASE, } \\
\text { um para professores e } \\
\text { outro para alunos, no } \\
\text { contexto escolar } \\
\text { (Emotional } \\
\text { Education } \\
\text { Programme for } \\
\text { Teachers) }\end{array}$ & $\begin{array}{l}\text { Programa para } \\
\text { desenvolvimento } \\
\text { de competências } \\
\text { socioemocionais }\end{array}$ & \begin{tabular}{|l|} 
Aumento das \\
competências \\
socioemocionais. \\
Melhora do clima em \\
sala de aula
\end{tabular} & $\begin{array}{l}30 \text { horas por } 9 \\
\text { meses }\end{array}$ \\
\hline 5 & \begin{tabular}{|l|} 
Jennings, Frank, \\
Snowberg, \\
Coccia, \& \\
Greenberg \\
(2013)
\end{tabular} & 50 docentes & $\begin{array}{l}\text { Avaliou o programa } \\
\text { CARE para ASE }\end{array}$ & $\begin{array}{l}\text { Programa de ASE } \\
\text { apoiado em } \\
\text { Mindfulness }\end{array}$ & $\begin{array}{l}\text { Aumento do bem-estar, } \\
\text { diminuição do estresse, } \\
\text { melhora da performance } \\
\text { e boa aceitação do } \\
\text { programa }\end{array}$ & $\begin{array}{l}5 \text { dias de } 6 \text { horas } \\
\text { ( } 30 \text { horas) ao } \\
\text { longo de } 3 \\
\text { meses, com } \\
\text { telefonemas de } \\
\text { coaching entre as } \\
\text { sessões e um } \\
\text { reforço ao final }\end{array}$ \\
\hline 6 & \begin{tabular}{|l} 
Fincias \& Izard \\
$(2013)$
\end{tabular} & $\begin{array}{l}\text { Não } \\
\text { especificado }\end{array}$ & $\begin{array}{l}\text { Avaliou um programa } \\
\text { de ASE }\end{array}$ & $\begin{array}{l}\text { Programa para } \\
\text { desenvolvimento } \\
\text { de competências } \\
\text { socioemocionais }\end{array}$ & \begin{tabular}{|l|} 
Melhora das \\
competências \\
socioemocionais de \\
professores do ensino \\
secundário
\end{tabular} & $\begin{array}{l}20 \text { horas de } \\
\text { treinamento } \\
\text { dividido em } 6 \\
\text { encontros ao } \\
\text { longo de } 2 \text { meses }\end{array}$ \\
\hline 7 & \begin{tabular}{|l|} 
Pérez-Escoda, \\
Guiu, Soldevila, \\
\& Fondevila \\
$(2013)$ \\
\end{tabular} & 92 docentes & $\begin{array}{l}\text { Avaliou um programa } \\
\text { de ASE }\end{array}$ & $\begin{array}{l}\text { Programa para } \\
\text { desenvolvimento } \\
\text { de competências } \\
\text { socioemocionais } \\
\end{array}$ & \begin{tabular}{|l|} 
Melhora das \\
competências \\
socioemocionais
\end{tabular} & 30 horas \\
\hline 8 & \begin{tabular}{|l|} 
Talvio, Lonka, \\
Komulainen, \\
Kuusela, \& \\
Lintunen (2013)
\end{tabular} & 69 docentes & $\begin{array}{l}\text { Avaliou o programa } \\
\text { Teacher Effectiveness } \\
\text { Training (TET) com } \\
\text { dois grupos de } \\
\text { professores }\end{array}$ & Programa de ASE & \begin{tabular}{|l|} 
Melhora das \\
competências \\
socioemocionais
\end{tabular} & $\begin{array}{l}\text { Programa TET - } \\
4 \text { dias de } \\
\text { treinamento, } 2 \text { no } \\
1^{\circ} \text { semestre, } 2 \text { no } \\
2^{\circ} \text { semestre }\end{array}$ \\
\hline 9 & $\begin{array}{l}\text { Karimzadeh, } \\
\text { Salehi, Embi, } \\
\text { Nasiri, \& } \\
\text { Shojaee (2014) }\end{array}$ & 68 docentes & $\begin{array}{l}\text { Avaliou um programa } \\
\text { de intervenção para } \\
\text { aumentar as } \\
\text { competências } \\
\text { socioemocionais em } \\
\text { professores }\end{array}$ & Programa de ASE & \begin{tabular}{|l|} 
Melhora das \\
competências \\
socioemocionais. \\
Melhora na eficácia de \\
ensino
\end{tabular} & $\begin{array}{l}20 \text { horas } \\
\text { divididas em } 10 \\
\text { encontros } \\
\text { semanais de } 2 \\
\text { horas }\end{array}$ \\
\hline 10 & \begin{tabular}{|l} 
Jennings, \\
Brown, Frank, \\
Tanler, Doyle, \\
Rasheed, ... \& \\
Greenberg \\
$(2014)$
\end{tabular} & 55 docentes & $\begin{array}{l}\text { Avaliou a replicação } \\
\text { do programa CARE } \\
\text { em professores de } 8 \\
\text { escolas }\end{array}$ & $\begin{array}{l}\text { Programa de ASE } \\
\text { apoiado em } \\
\text { Mindfulness }\end{array}$ & \begin{tabular}{|l|} 
Melhora do bem-estar. \\
Redução em sintomas \\
depressivos. Redução em \\
sintomas fisicos. \\
Diminuição do burnout. \\
Aumento do Mindfulness
\end{tabular} & $\begin{array}{l}30 \text { horas } \\
\text { divididas em } 4 \\
\text { dias inteiros } \\
\text { durante } 4-6 \\
\text { semanas, com } \\
\text { telefonemas de } \\
\text { coaching entre as } \\
\text { sessões e um } \\
\text { reforço no final }\end{array}$ \\
\hline
\end{tabular}




\begin{tabular}{|c|c|c|c|c|c|c|}
\hline 11 & $\begin{array}{l}\text { Wolf, Torrente, } \\
\text { Frisoli, } \\
\text { Weisenhorn, } \\
\text { Shivshanker, } \\
\text { Annan, \& Aber } \\
\text { (2015) }\end{array}$ & $\begin{array}{l}346 \\
\text { docentes }\end{array}$ & $\begin{array}{l}\text { Avaliou o programa } \\
\text { LRHC no bem-estar } \\
\text { de professores de } 64 \\
\text { escolas }\end{array}$ & Programa de ASE & $\begin{array}{l}\text { Aumento da insatisfação } \\
\text { entre professoras } \\
\text { mulheres e aumento da } \\
\text { motivação entre } \\
\text { professores menos } \\
\text { experientes }\end{array}$ & $\begin{array}{l}\text { LRHC (não foi } \\
\text { descrito) }\end{array}$ \\
\hline 12 & $\begin{array}{l}\text { Dolev \& } \\
\text { Leshem (2016) }\end{array}$ & 26 docentes & $\begin{array}{l}\text { Avaliou um programa } \\
\text { para o } \\
\text { desenvolvimento da } \\
\text { Inteligência } \\
\text { Emocional (IE) }\end{array}$ & $\begin{array}{l}\text { Programa de } \\
\text { desenvolvimento } \\
\text { de IE }\end{array}$ & $\begin{array}{l}\text { Aumento da IE, da } \\
\text { satisfação com o trabalho } \\
\text { docente e da relação com } \\
\text { os alunos }\end{array}$ & $\begin{array}{l}2 \text { anos, com } 12 \\
\text { encontros } \\
\text { mensais; no } 2^{\circ} \\
\text { ano, } 10 \\
\text { coachings } \\
\text { individuais e } \\
\text { workshops }\end{array}$ \\
\hline 13 & $\begin{array}{l}\text { Alexiou \& } \\
\text { Bouta (2016) }\end{array}$ & $\begin{array}{l}\text { Não } \\
\text { especificado }\end{array}$ & $\begin{array}{l}\text { Avaliou a aplicação } \\
\text { de um workshop } \\
\text { sobre ASE em } \\
\text { ambiente virtual } \\
\text { (Teachers' } \\
\text { Professional } \\
\text { Development } \\
\text { Program) } \\
\end{array}$ & $\begin{array}{l}\text { Aplicação de um } \\
\text { workshop sobre } \\
\text { ASE em ambiente } \\
\text { virtual }\end{array}$ & $\begin{array}{l}\text { Aumento da IE. Melhora } \\
\text { no reconhecimento dos } \\
\text { elementos da IE }\end{array}$ & $\begin{array}{l}\text { Workshop sobre } \\
\text { ASE em } \\
\text { ambiente virtual }\end{array}$ \\
\hline 14 & $\begin{array}{l}\text { Harvey, Evans, } \\
\text { Hill, } \\
\text { Henricksen, \& } \\
\text { Bimler (2016) }\end{array}$ & 27 docentes & $\begin{array}{l}\text { Avaliou um programa } \\
\text { voltado para a ASE }\end{array}$ & Programa de ASE & $\begin{array}{l}\text { Não houve relação entre } \\
\text { a mudança dos } \\
\text { professores e o } \\
\text { comportamento pro- } \\
\text { social dos alunos. } \\
\text { Alunos relataram } \\
\text { perceber mudança na } \\
\text { liderança e amistosidade } \\
\text { dos professores }\end{array}$ & $\begin{array}{l}\text { dias de } \\
\text { workshops, com } \\
\text { intervalo de } 3 \\
\text { semanas, mais } \\
\text { meio periodo de } \\
\text { acompanhamento } \\
\text { (total de } 3 \\
\text { meses) }\end{array}$ \\
\hline 15 & $\begin{array}{l}\text { Schussler, } \\
\text { Jennings, Sharp, } \\
\text { \& Frank (2016) }\end{array}$ & 50 docentes & $\begin{array}{l}\text { Avaliou programa } \\
\text { CARE para ASE }\end{array}$ & $\begin{array}{l}\text { Programa de ASE } \\
\text { apoiado em } \\
\text { Mindfulness }\end{array}$ & $\begin{array}{l}\text { Aumento no } \\
\text { autoconhecimento, } \\
\text { consciência somática, } \\
\text { aumento da necessidade } \\
\text { de autocuidado, } \\
\text { diminuição da } \\
\text { reatividade emocional. } \\
\text { Não se constatou } \\
\text { aumento da eficácia do } \\
\text { professor }\end{array}$ & $\begin{array}{l}30 \text { horas } \\
\text { distribuídas em } 4 \\
\text { dias inteiros num } \\
\text { espaço de } 6 \\
\text { semanas }+ \\
\text { telefonemas para } \\
\text { coaching entre as } \\
\text { sessões + reforço } \\
\text { no final }\end{array}$ \\
\hline 16 & $\begin{array}{l}\text { Dolev \& } \\
\text { Leshem (2017) }\end{array}$ & 21 docentes & $\begin{array}{l}\text { Avaliou um } \\
\text { treinamento de } \\
\text { Inteligência } \\
\text { Emocional }\end{array}$ & $\begin{array}{l}\text { Treinamento de } \\
\text { IE }\end{array}$ & $\begin{array}{l}\text { Melhora em } \\
\text { comportamentos } \\
\text { relacionados na vida } \\
\text { pessoal e profissional }\end{array}$ & $\begin{array}{l}\text { 12 workshops } \\
\text { com intervalo de } \\
3-4 \text { semanas e } 10 \\
\text { sessões de } \\
\text { coaching } \\
\text { individuais com } \\
\text { intervalo de 2-3 } \\
\text { semanas }\end{array}$ \\
\hline 17 & $\begin{array}{l}\text { Jennings, } \\
\text { Brown, Frank, } \\
\text { Doyle, Oh, } \\
\text { Davis, ... \& } \\
\text { Greenberg } \\
(2017)\end{array}$ & $\begin{array}{l}224 \\
\text { docentes }\end{array}$ & $\begin{array}{l}\text { Avaliou a eficácia do } \\
\text { programa CARE para } \\
\text { a ASE em } \\
\text { professores de } 36 \\
\text { escolas }\end{array}$ & $\begin{array}{l}\text { Programa de ASE } \\
\text { apoiado em } \\
\text { Mindfulness }\end{array}$ & $\begin{array}{l}\text { Aumento das } \\
\text { competências } \\
\text { socioemocionais. } \\
\text { Melhora da qualidade } \\
\text { das interações }\end{array}$ & $\begin{array}{l}5 \text { dias de } 6 \text { horas } \\
\text { ( } 30 \text { horas) em } 3 \\
\text { meses, com } \\
\text { telefonemas de } \\
\text { coaching entre as } \\
\text { sessões e um } \\
\text { reforço ao final }\end{array}$ \\
\hline 18 & $\begin{array}{l}\text { Castillo-Gualda, } \\
\text { García, Pena, } \\
\text { Galán, \& } \\
\text { Brackett (2017) }\end{array}$ & 54 docentes & $\begin{array}{l}\text { Avaliou o programa } \\
\text { RULER }\end{array}$ & $\begin{array}{l}\text { Programa para o } \\
\text { desenvolvimento } \\
\text { da IE }\end{array}$ & $\begin{array}{l}\text { Aumento da percepção } \\
\text { emocional,compreensão, } \\
\text { regulação emocional, } \\
\text { satisfação e } \\
\text { comprometimento com o } \\
\text { trabalho }\end{array}$ & $\begin{array}{l}8 \text { sessões de } 3 \\
\text { horas ( } 24 \text { horas) } \\
\text { em } 3 \text { meses }\end{array}$ \\
\hline
\end{tabular}


TA B EL A 2

Estudos Encontrados a Partir da Combinação dos Descritores Teacher, Social and Emotional or Emotional Intelligence, Program or Intervention, School

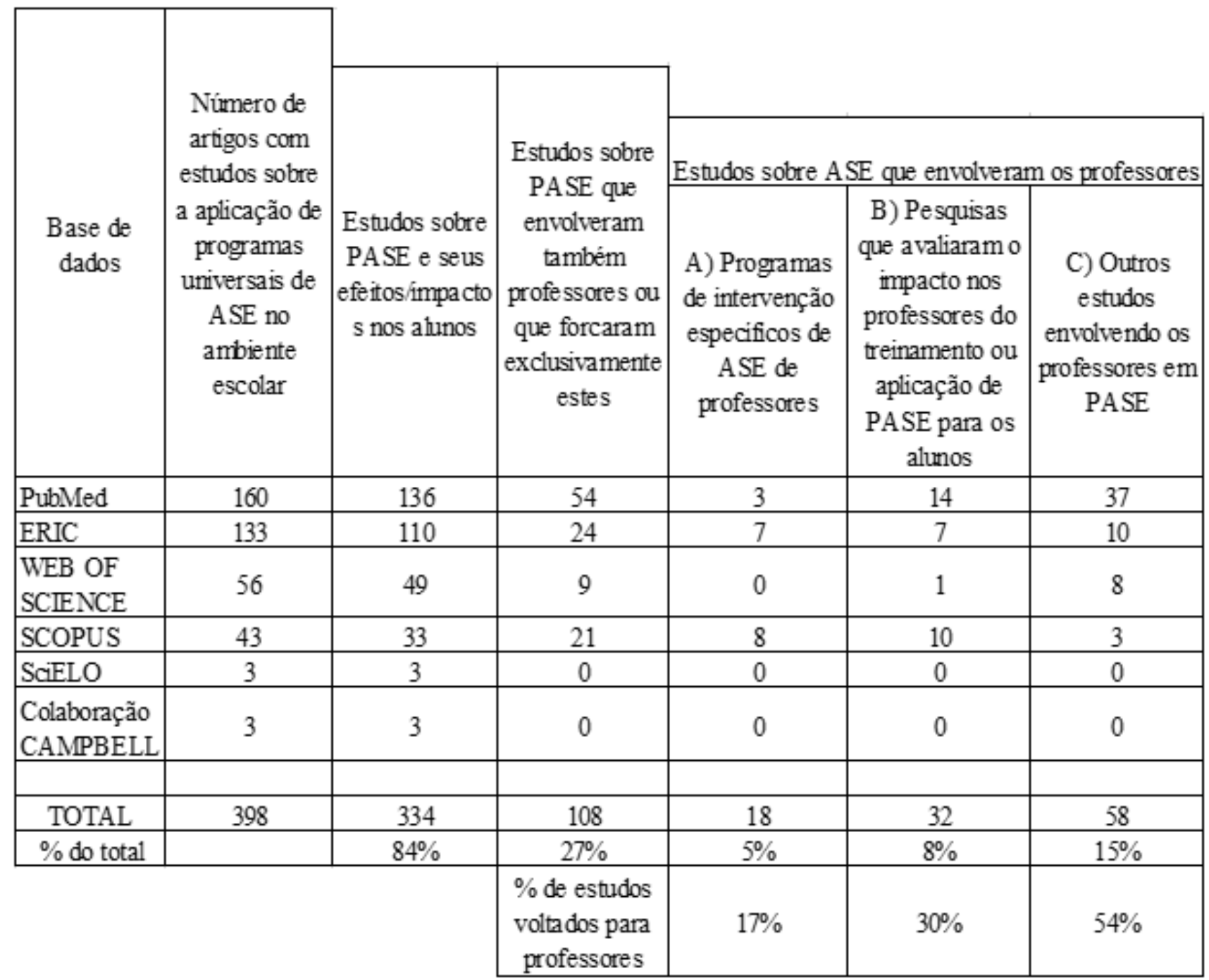

Outro grupo de artigos (58 artigos - 15\%) envolve o professor em aspectos relacionados às intervenções ou PASEs na escola numa relação menos direta com sua própria competência nesta dimensão (Esen-Aygun \& Sahin-Taskin, 2017; Paxton, Wanless, \& Rimm-Kaufman, 2013; Shek \& Chan, 2011; Shek \& Sun, 2013; Shervey, Sandilos, DiPerna \& Lei, 2017). Estes estudos têm o professor como participante de algum modo, mas não tratam de sua própria competência neste aspecto (Tabela 2).

Do total encontrado a partir do critério de busca, somente 18 artigos tiveram como objetivo específico programas de intervenção para desenvolver as habilidades socioemocionais do professor e foram selecionados para análise (Tabela 2). Destes, oito artigos foram encontrados na base de dados SCOPUS, sete artigos na base ERIC e três artigos foram encontrados na base de dados PubMed. Entre os países que realizaram os estudos, houve a predominância de pesquisas nos Estados Unidos, com oito artigos (44\%), seguidos pela Espanha, com quatro estudos (22\%), Iran e Israel, com dois artigos cada (11\% cada país), e Finlândia, Grécia e Nova 
TA B E L A 3

Características dos Programas de Intervenção para ASE para Professores

\begin{tabular}{|c|c|c|c|}
\hline $\begin{array}{c}\text { Programas de intervenção de ASE } \\
\text { para professores }\end{array}$ & $\begin{array}{c}\text { Número } \\
\text { de artigos }\end{array}$ & $\begin{array}{l}\text { No. do } \\
\text { estudo }\end{array}$ & Características \\
\hline $\begin{array}{l}\text { CARE - Cultivating Awareness and } \\
\text { Resilience in Education }\end{array}$ & 5 & $\begin{array}{c}\text { Estudos 2, } \\
5,10,15 \mathrm{e} \\
17\end{array}$ & $\begin{array}{l}30 \text { horas presenciais divididas em 4-6 } \\
\text { dias durante } 4-6 \text { semanas, com } \\
\text { telefonemas de coaching entre as } \\
\text { sessões e um reforço no final. }\end{array}$ \\
\hline $\begin{array}{l}\text { CEBT - Cultivating Emotional Balance } \\
\text { Training }\end{array}$ & 1 & Estudo 1 & $\begin{array}{l}8 \text { semanas (encontros presenciais em } 4 \\
\text { tardes e } 4 \text { dias inteiros) envolvendo } \\
\text { conteúdo teórico e práticas de } \\
\text { Mindfulness }\end{array}$ \\
\hline $\begin{array}{l}\text { Programa EIT - Emotional } \\
\text { Intelligence Training }\end{array}$ & 1 & Estudo 8 & $\begin{array}{l}4 \text { dias de treinamento presencial, } 2 \text { no } \\
\text { 1o. semestre, } 2 \text { no } 20 \text {. semestre }\end{array}$ \\
\hline $\begin{array}{l}\text { LRHC - Learning to Read in a Healing } \\
\text { Classroom }\end{array}$ & 1 & Estudo 11 & Não foi descrito \\
\hline $\begin{array}{l}\text { Programa EIT - Emotional Intelligence } \\
\text { Trainning }\end{array}$ & 1 & Estudo 3 & 10 sessões semanais de 2 horas. \\
\hline Programa RULER & 1 & Estudo 18 & $\begin{array}{l}24 \text { horas em três meses ( } 8 \text { sessõ es de } \\
3 \text { horas) }\end{array}$ \\
\hline $\begin{array}{l}\text { Karimzadeh, Salehi, Embi, Nasiri \& } \\
\text { Shojaee (2014) } \\
\text { (Programa sem nomenclatura) }\end{array}$ & 1 & Estudo 9 & $\begin{array}{l}20 \text { horas divididas em } 10 \text { encontros } \\
\text { semanais de } 2 \text { horas. }\end{array}$ \\
\hline $\begin{array}{l}\text { Dolev \& Leshem (2016) } \\
\text { Dolev \& Leshem (2017) } \\
\text { sem nomenclatura) }\end{array}$ & 2 & $\begin{array}{c}\text { Estudo } 12 \mathrm{e} \\
16\end{array}$ & $\begin{array}{l}\text { Programa com duração de } 2 \text { anos. No } \\
\text { primeiro, } 12 \text { encontros mensais com } \\
\text { duração de poucas horas a um dia } \\
\text { todo. No } 2^{\circ} \text { ano, } 10 \text { coachings } \\
\text { individuais a cada } 2 \text { ou } 3 \text { semanas, } \\
\text { acompanhado de algumas oficinas. }\end{array}$ \\
\hline $\begin{array}{l}\text { Harvey, Evans, Hill, Henricksen \& } \\
\text { Bimler (2016) } \\
\text { (Programa sem nomenclatura) }\end{array}$ & 1 & Estudo 14 & $\begin{array}{l}3 \text { dias de oficinas, com intervalo de } 3 \\
\text { semanas, mais meio periodo de } \\
\text { acompanhamento (total de } 3 \text { meses). }\end{array}$ \\
\hline $\begin{array}{l}\text { Pérez-Escoda, Guiu, Soldevila \& } \\
\text { Fondevila (2013) } \\
\text { (Programa sem nomenclatura) }\end{array}$ & 1 & Estudo 7 & 30 horas \\
\hline $\begin{array}{l}\text { Fincias \& Izard (2013) } \\
\text { (Programa sem nomenclatura) }\end{array}$ & 1 & Estudo 6 & $\begin{array}{l}20 \text { horas de treinamento dividido em } 6 \\
\text { encontros ao longo de } 2 \text { meses }\end{array}$ \\
\hline $\begin{array}{l}\text { Emotional Education Programme for } \\
\text { teachers }\end{array}$ & 1 & Estudo 4 & 30 horas por 9 meses \\
\hline $\begin{array}{l}\text { Teachers' Pro fessional Development } \\
\text { programs (TPD) }\end{array}$ & 1 & Estudo 13 & ASE em ambiente virtual. \\
\hline
\end{tabular}


Zelândia, com um artigo cada (6\% cada país). Na busca realizada, não houve delimitação de período. O primeiro estudo encontrado sobre PASE específicos para o professor data de 2011, o que evidencia que o interesse pelo tema é recente. Dos 18 estudos selecionados, em 14 deles foram usados métodos quantitativos de pesquisa $(78 \%)$, dois artigos apresentaram estudos com métodos qualitativos (11\%) e dois artigos envolveram pesquisas com métodos mistos (11\%).

Analisando os estudos encontrados, constatase que não há uma uniformidade entre as intervenções ou programas voltados para a aprendizagem socioemocional do docente. Os tipos de PASE voltados para professores são bastante variados quanto a sua duração, frequência e mesmo estratégias, conforme apresentado na Tabela 3.

A maioria dos programas de intervenção envolve encontros presenciais em grupo na forma de treinamento ou oficina (12 programas/intervenções - $\quad 86 \%$ ). Dois envolveram também estratégias contemplativas para o desenvolvimento das habilidades socioemocionais (Mindfulness - 14\%); dois programas incluíram sessões individuais de orientação (coaching - 11\%); um programa incluiu ligações telefônicas para orientação (coaching por telefone - 7\%); e um programa foi feito a partir de ambiente virtual (7\%).

O maior uso nos programas de intervenção de atividades presenciais vai ao encontro do entendimentodeque desenvolver competências que envolvem habilidades emocionais é um tipo de aprendizagem que difere, em certa medida, da aprendizagem convencional de fatos, de conceitos ou de procedimentos. Como a dimensão emocional envolve aspectos inconscientes e involuntários, trazer alguns destes componentes à consciência demanda um método que envolva entrar em contato com as emoções e vivenciá-las, sendo seguido de reflexões e trocas entre os participantes no grupo ou com o facilitador.

A totalidade dos estudos encontrados evidenciou efeitos ou impactos positivos em algum aspecto relacionado às competências socioemocionais, ao bem-estar do professor, em sua eficácia de ensino ou ao clima da sala de aula, conforme a Tabela 4 .

Estes dados tornam evidente que PASEs para o professor geram benefícios, não somente no que tange ao aumento de suas competências socioemocionais como também em relação a sua atuação como educador e a sua saúde e bem-estar, o que pode ter reflexos importantes na educação escolar como um todo. No entanto, em razão do baixo número de estudos voltados a esta temática, a abrangência dos resultados é limitada.

Vale ressaltar que mesmo incluindo em todas as buscas a palavra "professor", dos 398 artigos inicialmente identificados sobre a aplicação de programas de intervenção em aprendizagem socioemocional na escola, apenas 5\% (18 artigos) se referiam a pesquisas voltadas especificamente para o desenvolvimento das competências socioemocionais do professor. Estes números indicam que há ainda pouco interesse sobre PASEs para o professor como uma maneira de aumentar sua competência nesta área, ampliar seus recursos para lidar com as demandas do trabalho e na promoção de seu bem-estar, assim como parece haver pouco interesse na investigação sobre o desenvolvimento das competências socioemocionais do professor como um caminho para a melhora da qualidade do ensino. Uma das justificações possíveis para o pouco interesse da comunidade científica neste sentido é existir a crença de que o professor já tem estas competências - ou deveria tê-las. Outra hipótese para o baixo número de estudos sobre o tema é a crença de que um adulto não deveria ser sujeito a um processo educativo 
TA B EL A 4

Impactos ou efeitos no professor dos programas de intervenção para ASE

\begin{tabular}{|c|c|r|}
\hline $\begin{array}{c}\text { Impactos e efeitos das } \\
\text { intervenções ou programas de } \\
\text { ASE nos professores }\end{array}$ & $\begin{array}{c}\text { Número de } \\
\text { artigos }\end{array}$ & $* \%$ \\
\hline $\begin{array}{c}\text { Melhora nas Habilidades } \\
\text { Socioemocionais }\end{array}$ & 12 & $67 \%$ \\
\hline Aumento do bem-estar & 8 & $44 \%$ \\
\hline Diminuição no estresse & 4 & $22 \%$ \\
\hline Melhora no clima escolar & 5 & $28 \%$ \\
\hline Melhora da eficácia do ensino & 3 & $17 \%$ \\
\hline Diminuição de sintomas fisicos & 2 & $11 \%$ \\
\hline TOTAL & $\mathbf{1 8}$ & \\
\hline
\end{tabular}

* Um mesmo estudo pode ter apresentado mais de um efeito ou impacto nos professores.

que envolva emoções e comportamentos.

\section{CONCLUS Ão}

Várias pesquisas têm confirmado que os professores são expostos a alto nível de estresse e de tensão ( Gomes \& Quintão, 2011; Harris, Jennings, Katz, Abenavoli, \& Greenberg, (2016); Herman, Hickmon-Rosa, \& Reinke, 2017). O professor sente-se pressionado ao assumir papéis sociais múltiplos, o que gera tensão entre a necessidade de respostas e os recursos de que dispõe (Gomes \& Pereira, 2008).

O uso de estratégias de regulação emocional para lidar com as situações estressantes e desenvolver melhores formas para manejá-las tem se mostrado efetivo na promoção do bemestar psicológico e na redução do estresse em grupos expostos ao estresse crônico, como é o caso do professor. Estas estratégias auxiliam tanto no manejo interno das emoções como também favorecem que se consiga controlar, em alguma medida, os eventos externos. A capacidade de regulação emocional está também diretamente relacionada às habilidades sociais (Andreotti, 2013).

Com a crescente evidência de que ações intencionais para o aprendizado socioemocional do aluno podem favorecer seu desempenho acadêmico, muitos professores são instados a promover este ensino em sala de aula. Mas, aparentemente, assume-se que o professor já tem (ou deveria ter) competências socioemocionais suficientes, fazendo com que o mesmo conte apenas com as que já possui, uma vez que, na grande maioria das vezes, esta temática não é contemplada em sua formação inicial ou continuada (Zinsser, Christensen, \& Torres, 2016).

Há evidências de que professores com menos competências socioemocionais têm mais dificuldade em lidar com suas emoções. Estudos relacionam estes professores a alunos que experimentam mais emoções negativas na sala de aula, o que pode afetar o nível de motivação e engajamento do discente com o aprendizado (Karimzadeh et al., 2014). Por outro lado, professores com mais competências socioemocionais mostram-se mais eficientes 
no ensino, compreendem melhor as emoções de seus alunos e são mais capazes de dar suporte em momentos emocionalmente difíceis; lidam e manejam melhor o comportamento dos estudantes em sala de aula e são mais competentes em desenvolver competências socioemocionais nos alunos. Estes aspectos favorecem a criação de um clima saudável em sala de aula que contribui para o sucesso acadêmico, o que pode aumentar a satisfação do professor com seu trabalho e diminuir o estresse, gerando um ciclo virtuoso.

A maioria dos estudos encontrados nesta revisão integrativa da literatura que envolveu o professor e a aprendizagem socioemocional volta-se para a percepção do docente sobre programas para aprendizagem socioemocional que têm como alvo os alunos e a qualidade e fidelidade do professor em sua implementação, assim como diferentes formas de treinamento para que o professor possa aplicá-los. Apenas 5\% dos estudos (18 estudos) encontrados contemplaram programas voltados especificamente para o professor. E, embora estes 18 estudos tenham envolvido diferentes metodologias para a ASE, a totalidade deles evidenciou efeitos ou impactos positivos no bem-estar, na saúde ou na eficácia do professor em sua prática de ensino.

A docência é caracterizada como uma atividade profissional altamente estressante, com impactos importantes na saúde e na qualidade do ensino do professor. Quando faltam recursos ao educador para lidar com os desafios sociais e emocionais no contexto da sala de aula, além do aumento de seu nível de estresse, pode haver a deterioração do clima emocional e a piora no desempenho dos alunos. Parece então injustificado o fato de que haja, até ao momento, pouco interesse da comunidade científica por pesquisas que se voltem à aprendizagem socioemocional do docente. Assim, mostra-se fundamental que a aprendizagem socioemocional faça parte da formação inicial e continuada do docente. A maior complexidade encontrada nas sociedades, que também se reflete no ambiente escolar, demanda uma formação docente que inclua o desenvolvimento de novas competências, entre elas emocionais e sociais, fazendo com que o educador esteja melhor preparado para executar com êxito sua atividade profissional.

Do mesmo modo, é essencial a investigação sobre ações que possam promover o desenvolvimento da competência socioemocional do professor como um recurso que pode favorecer o bem-estar do mesmo, ter impactos positivos em sua saúde física e mental, assim como contribuir para a melhora da qualidade do ensino nas escolas brasileiras.

\section{REFERÊ NCIAS}

As referências marcadas com asterisco indicam estudos incluídos na revisão integrativa

${ }^{\star}$ Alexiou, A., \& Bouta, H. (2016, July). A case of emotional intelligence for teachers' professional development: Emotions and connections are ubiquitous in second life. In L. Uden, D. Liberona, \& B. Feldmann (Eds.), Learning Technology for Education in CloudThe Changing Face of Education. Proceedings of the 5th International Workshop, LTEC 2016 (Vol. 620, pp. 39-50). Heidelberg: Springer.

Andreotti, C. (2013). Effects of acute and chronic stress on attention and psychobiological stress reactivity in women (Doctoral dissertation). Vanderbilt University, E.U.A.

Bonotto, G., \& Felicetti, V. L. (2014). Habilidades e competências na prática docente: Perspectivas a partir de situações- 
problema. Educação por escrito, 5(1), 17-29. Carlotto, M. S. (2011). The burnout syndrome in teachers: Prevalence and associated factors. Psicologia: Teoria e Pesquisa, 27(4), 403-410.

CASEL. (2019, janeiro 3). What is social and emotional learning? [Página Web]. Recuperado de: https://casel.org

*Castillo-Gualda, R., García, V., Pena, M., Galán, A., \& Brackett, M. A. (2017). Resultados preliminares del método RULER en la inteligencia emocional y el compromiso laboral de profesores Españoles. Electronic Journal of Research in Education Psychology, 15(43), 641-664.

Damásio, A. (2012). O erro de Descartes: Emoção, razão e o cérebro humano. São Paulo: Editora Companhia das Letras.

D’Avila, O. P., Pinto, L. F. S., Hauser, L., Gonçalves, M. R., \& Harzheim, E. (2017). $\mathrm{O}$ uso do Primary Care Assessment Tool (PCAT): Uma revisão integrativa e proposta de atualização. Ciência \& Saúde Coletiva, 22(3), 855-865. https://doi. org/10.1590/1413-81232017223.03312016

${ }^{\star}$ Dolev, N., \& Leshem, S. (2016). Teachers' emotional intelligence: The impact of training. CRES Special Issue, 8(1), 75-94.

${ }^{\star}$ Dolev, N., \& Leshem, S. (2017). Developing emotional intelligence competence among teachers. Teacher Development, 21(1), 2139. https://doi.org/10.1080/13664530.2016. $\underline{1207093}$

Dutra, J. S., Hipólito, J. A. M., \& Silva, C. M. (2000). Gestão de pessoas por competências: $\mathrm{O}$ caso de uma empresa do setor de telecomunicações. Revista de Administração Contemporânea, 4(1), 161-176.

Esen-Aygun, H., \& Sahin-Taskin, C. (2017). Teachers' views of social-emotional skills and their perspectives on social-emotional learning programs. Journal of Education and Practice, 8(7), 205-215.
Fernet, C., Guay, F., Senécal, C., \& Austin, S. (2012). Predicting intraindividual changes in teacher burnout: The role of perceived school environment and motivational factors. Teaching and Teacher Education, 28(4), 514-525. https://doi.org/10.1016/j. tate.2011.11.013

Feuerhahn, N., Bellingrath, S., \& Kudielka, B. M. (2013). The interplay of matching and non-matching job demands and resources on emotional exhaustion among teachers. Applied Psychology: Health and Well-Being, 5(2), 171-192. https://doi.org/10.1111/ aphw.12002

*Fincias, P. T., \& Martín Izard, J. F. (2013, November). Development and evaluation of emotional competences in secondary education teachers: Design of an applied research. In F. J. García-Peñalvo (Ed.), First International Conference on Technological Ecosystem for Enhancing Multiculturality (TEEM'13), Salamanca, Spain, (pp. 591595). Salamanca: ACM.

Fisher, M. H. (2011). Factors influencing stress, burnout, and retention of secondary teachers. Current Issues in Education, 14(1), $1-36$.

Gomes, A. P. R., \& Quintão, S. R. (2011). Burnout, satisfação com a vida, depressão e carga horária em professores. Análise Psicológica, 29(2), 335-344.

Gomes, R., \& Pereira, A. (2008). Estratégias de coping em educadores de infância portugueses. Psicologia Escolar $e$ Educacional, 12(2), 319-326. http://dx.doi. org/10.1590/S1413-85572008000200003

Gómez-Gascón, T., Martín-Fernández, J., Gálvez-Herrer, M., Tapias-Merino, E., Beamud-Lagos, M., Mingote-Adán, J. C., \& EDESPROAP-Madrid, G. (2013). Effectiveness of an intervention for prevention and treatment of burnout in primary health care professionals. $B M C$ 
Family Practice, 1(14), 1-7.

Harris, A. R., Jennings, P. A., Katz, D. A., Abenavoli, R. M., \& Greenberg, M. T. (2016). Promoting stress management and wellbeing in educators: Feasibility and efficacy of a school-based Yoga and Mindfulness intervention. Mindfulness, 7(1), 143-154. https://doi.org/10.1007/s12671-015-0451-2

${ }^{*}$ Harvey, S. T., Evans, I. M., Hill, R. V. J., Henricksen, A., \& Bimler, D. (2016). Warming the emotional climate of the classroom: Can teachers' social-emotional skills change?. International Journal of Emotional Education, 8(2), 70-87.

Herman, K. C., Hickmon-Rosa, J. E., \& Reinke, W. M. (2017). Empirically derived profiles of teacher stress, burnout, self-efficacy, and coping and associated student outcomes. Journal of Positive Behavior Interventions, 20(2), 90-100.

Jennings, P. A., \& Greenberg, M. T. (2009). The prosocial classroom: Teacher social and emotional competence in relation to student and classroom outcomes. Source: Review of Educational Research, 79(1), 491-525. https://doi.org/10.3102/0034654308325693

${ }^{\star}$ Jennings, P. A., Brown, J. L., Frank, J. L., Doyle, S., Oh, Y., Davis, R., ... Greenberg, M. T. (2017). Impacts of the CARE for teachers program on teachers' social and emotional competence and classroom interactions. Journal of Educational Psychology, 109(7), 1010-1028. $\quad$ https://doi.org/10.1037/ $\underline{\text { edu0000187 }}$

*Jennings, P. A., Brown, J. L., Frank, J., Tanler, R., Doyle, S., Rasheed, D., ... Greenberg, M. (2014). Promoting teachers' social and emotional competence: A replication study of the cultivating awareness and resilience in education (CARE) program. SREE Spring 2014 Conference, Society for Research on Educational Effectiveness. Recuperado de: https://eric.ed.gov/?id=ED562749
${ }^{\star} J e n n i n g s$, P. A., Foltz, C., Snowberg, K. E., Sim, H., \& Kemeny, M. E. (2011). The influence of mindfulness and emotion skills training on teachers' classrooms: The effects of the cultivating emotional balance training. Recuperado de: https://eric. ed.gov/?id=ED518584

*Jennings, P. A., Frank, J. L., Snowberg, K. E., Coccia, M. A., \& Greenberg, M. T. (2013). Improving classroom learning environments by cultivating awareness and resilience in education (CARE): Results of a randomized controlled trial. School Psychology Quarterly, 28(4), 374-390. https://doi.org/10.1037/ spq0000035

${ }^{\star}$ Jennings, P. A., Snowberg, K., Coccia, M., \& Greenberg, M. (2011). Improving classroom learning environments by cultivating awareness and resilience in education (CARE): Results of two pilot studies. Journal of Classroom Interaction, 46, 37-48. http:// www.jstor.org/stable/23870550.

Jones, S. M., Bouffard, S. M., \& Weissbourd, R. (2013). Educators' social and emotional skills vital to learning. Phi Delta Kappan, 94(8), 62-65. https://doi. org/10.1177/003172171309400815

*Karimzadeh, M., Goodarzi, A., \& Rezaei, S. (2012). The effect of social emotional skills training to enhance general health \& emotional intelligence in the primary teachers. Procedia - Social and Behavioral Sciences, 46, 57-64. https://doi.org/10.1016/j. sbspro.2012.05.068

${ }^{\star}$ Karimzadeh, M., Salehi, H., Embi, M. A., Nasiri, M., \& Shojaee, M. (2014). Teaching efficacy in the classroom: Skill based training for teachers' empowerment. English Language Teaching, 7(8), 106-115. https:// doi.org/10.5539/elt.v7n8p106

Marin, A. H., Silva, C. T. D., Andrade, E. I. D., Bernardes, J., \& Fava, D. C. (2017). Competência socioemocional: Conceitos e 
instrumentos associados. Revista Brasileira de Terapias Cognitivas, 13(2), 92-103.

Martinsone, B., \& Damberga, I. (2017). Qualitative analysis of teachers' written selfreflections after implementation of a socialemotional learning program in Latvia. International Journal of School \& Educational Psychology, 5(4), 215-225. https://doi.org/1 $\underline{0.1080 / 21683603.2016 .1225236}$

Maslach, C., Schaufeli, W. B., \& Leiter, M. P. (2001). Job burnout. Annual Review of Psychology, 52, 397-422. https://doi. org/10.1146/annurev.psych.52.1.397

Maulana, R., Opdenakker, M. C., \& Bosker, R. (2014). Teacher-student interpersonal relationships do change and affect academic motivation: A multilevel growth curve modelling. British Journal of Educational Psychology, 84(3), 459-482. https://doi. org/10.1111/bjep.12031

Maulana, R., Opdenakker, M. C., Stroet, K., \& Bosker, R. (2013). Changes in teachers' involvement versus rejection and links with academic motivation during the first year of secondary education: A multilevel growth curve analysis. Journal of Youth and Adolescence, 42(9), 1348-1371. https://doi. org/10.1007/s10964-013-9921-9

Paxton, C. L. C., Wanless, S. B., \& RimmKaufman, S. E. (2013). Coaching support, context, and fidelity of implementation in Responsive Classroom ${ }^{\circledR}$ schools. SREE Spring 2013 Conference, Society for Research on Educational Effectiveness, 1-4. https:// files.eric.ed.gov/fulltext/ED563070.pdf

*Pérez-Escoda, N., Filella, G., Alegre, A., \& Bisquerra, R. (2012). Developing the emotional competence of teachers and pupils in school contexts. Electronic Journal of Research in Educational Psychology, 10(3), 1183-1208.

*Pérez-Escoda, N., Guiu, G. F., Soldevila, A., \& Fondevila, A. (2013). Evaluación de un programa de educación emocional para profesorado de primaria. Educación XX1, 16(1), 233-254. https://doi.org/10.5944/ educxx1.16.1.725

${ }^{\star}$ Schussler, D. L., Jennings, P. A., Sharp, J. E., \& Frank, J. L. (2016). Improving teacher awareness and well-being through CARE: A qualitative analysis of the underlying mechanisms. Mindfulness, 7(1), 130-142. https://doi.org/10.1007/s12671-015-0422-7

Shek, D. T. L., \& Chan, C. (2011). Qualitative evaluation of the Secondary 3 Training Program of the Project P.A.T.H.S. in Hong Kong. International Journal of Adolescent Medicine and Health, 23(4), 351-356. https://doi.org/10.1515/IJAMH.2011.032

Shek, D. T. L., \& Sun, R. C. F. (2013). Evaluation of project P.A.T.H.S. by the program implementers: Findings based on the extension phase. International Journal of Adolescent Medicine and Health, 25(4), 415-423. https://doi.org/10.1515/ ijamh-2013-0039

Shervey, S. W., Sandilos, L. E., DiPerna, J. C., \& Lei, P. W. (2017). Social validity of the social skills improvement system - Classwide intervention program (SSIS-CIP) in the primary grades. Grantee Submission, 32(3), 414-421.

Souza, M. T., Silva, M. D., \& Carvalho, R. (2010). Integrative review: What is it? How to do it? Einstein (São Paulo), 8(1), 102-106. https:// doi.org/10.1590/S1679-45082010RW1134

Steinhardt, M. A., Smith Jaggars, S. E., Faulk, K. E., \& Gloria, C. T. (2011). Chronic work stress and depressive symptoms: Assessing the mediating role of teacher burnout. Stress and Health, 27(5), 420-429. https://doi. org/10.1002/smi.1394

*Talvio, M., Lonka, K., Komulainen, E., Kuusela, M., \& Lintunen, T. (2013). Revisiting Gordon's teacher effectiveness training: An intervention study on teachers' social and 
emotional learning. Electronic Journal of Research in Educational Psychology, 11(3), 693-716. https://core.ac.uk/download/ pdf/143455545.pdf

Wang, M., \& Saudino, K. J. (2011). Emotion regulation and stress. Journal of Adult Development, 18(2), 95-103. https://doi. org/10.1007/s10804-010-9114-7

${ }^{\star}$ Wolf, S., Torrente, C., Frisoli, P., Weisenhorn, N., Shivshanker, A., Annan, J., \& Aber, J. L. (2015). Preliminary impacts of the "Learning to Read in a Healing Classroom" intervention on teacher well-being in the Democratic Republic of the Congo. Teaching and Teacher Education, 52, 24-36. https:// doi.org/10.1016/j.tate.2015.08.002

Zinsser, K. M., Christensen, C. G., \& Torres, L. (2016). She's supporting them; who's supporting her? Preschool center-level social-emotional supports and teacher wellbeing. Journal of School Psychology, 59, 5566. https://doi.org/10.1016/j.jsp.2016.09.001

\section{EVALUATION OF INTERVENTION PROGRAMS FOR TEACHER'S SOCIAL AND EMOTIONAL LEARNING: AN INTEGRATIVEREVIEW}

\section{Abstract}

The teaching profession generates great emotional demand, which can affect the well-being and generate health problems for teachers. Socioemotional skills involve a set of skills, knowledge and attitudes to better recognize and cope with one's own emotions and the emotional dimension of the students, which can decrease stress and increase the quality of teacher practice. Social-emotional learning can increase internal resources and the competence to better handle professional demands. In this integrative literature review, we searched the main world databases (SCOPUS, PubMed, ERIC, SciELO, Web of Science, Campbell Collaboration) for studies on specific intervention programs to develop socio-emotional skills of the school teacher, their characteristics and impacts or effects on the teacher. We included 18 articles on studies that aimed at the social-emotional learning of the teacher her/himself, not associated with programs aimed at students. It was concluded that although there are few studies on the subject, all had positive impacts or effects in the increase of social-emotional skills, in health, well-being, or in teaching effectiveness.

KEYwORDs: Teacher; Social and emotional learning; Stress; Integrative review 
EVALUACIÓN DE PROGRAMAS DE INTERVENCIÓN PARA EL APRENDIZAJE SOCIOEMOCIONAL DEL PROFESOR: UNA REVISIÓN INTEGRATIVA

\section{RESUMEN}

La profesión docente genera una gran demanda emocional, lo que puede afectar el bienestar del profesor y generar problemas de salud. Las competencias socioemocionales involucran un conjunto de habilidades, conocimientos y actitudes para reconocer $y$ lidiar mejor con las propias emociones y con la dimensión emocional de los alumnos, lo que puede disminuir el estrés y aumentar la calidad de la práctica del profesor. El aprendizaje socioemocional puede aumentar los recursos internos y la competencia para lidiar mejor con las demandas profesionales. En esta revisión integrativa de la literatura se buscó levantar en las principales bases de datos mundiales (SCOPUS, PubMed, ERIC, SciELO, Web of Science, Campbell Collaboration) los estudios realizados sobre programas de intervención específicos para desarrollar las competencias socioemocionales del profesor de enseñanza básica, sus características y impactos o efectos en el profesor. Se incluyeron 18 artículos sobre estudios que tuvieron como objetivo el aprendizaje socioemocional del propio profesor, no asociados a programas dirigidos a los alumnos. Se concluyó que aunque hay pocos estudios sobre el tema, todos presentaron impactos o efectos positivos en el aumento de habilidades socioemocionales, en la salud, bienestar, o en la eficacia docente.

Palabras Clave: Profesor; Aprendizaje socioemocional; Estrés; Revisión integrativa

\footnotetext{
${ }^{\text {I }}$ Programa de Pós-Graduação da Escola Paulista de Enfermagem - EPE, Universidade Federal de São Paulo - UNIFESP, Brasil. ORCID: 0000-0002-9557-9937

${ }^{\text {II }}$ Departamento de Administração e Saúde Coletiva, Programa de Pós-Graduação em Enfermagem, Universidade Federal de São Paulo - UNIFESP, Brasil. ORCID: 0000-0003-4344-1116

II Programa de Pós-Graduação do Departamento de Psiquiatria e Psicologia Médica, Escola Paulista de Medicina, Universidade Federal de São Paulo - UNIFESP, Brasil. ORCID 0000-0003-0237-6610
} 